\title{
Health benefits of joint replacement surgery for patients with osteoarthritis: prospective evaluation using independent assessments in Scotland
}

\author{
Sheina Orbell, Arthur Espley, Marie Johnston, David Rowley
}

\begin{abstract}
Study objectives-To determine extent of change in psychological, functional, and social health after knee and hip joint replacement surgery using independent assessments.

Design-Patients were recruited before surgery and interviewed preoperatively, three months after surgery, and nine months after surgery. Interviews were conducted in the patients' own homes.

Setting-Two orthopaedic surgery units in Scotland.

Participants-A consecutive sample of 107 patients with osteoarthritis having primary replacement of the knee or hip.

Main outcome measures-Assessments of depression, anxiety, pain, functional activity, informal care, and formal service utilisation were made at three time points. Main results-Anxiety and pain were significantly reduced and functional activity levels significantly increased after surgery. While gains in anxiety and pain reduction occurred between the preoperative and three month assessments, gains in activity were made between the three month and nine month assessments. Although pain was reduced and activity increased, levels of depression were unchanged after surgery. Patients reported need for assistance with fewer activities after surgery, but increases in the use of formal services and increases in the number of hours per week of informal support received were observed at both three month and nine month follow up. Conclusions-The main benefit of joint replacement surgery is pain relief. Gains in functional activity, particularly mobility and leisure activities are made by many patients. Paradoxically, surgery for osteoarthritis seems to act as a "gateway" to increases in formal and informal community support, which are maintained into the longer term.

(f Epidemiol Community Health 1998;52:564-570)
\end{abstract}

Arthritis represents a significant threat to public health and a considerable cause of disability and dependency, particularly among older people. In the absence of effective primary or secondary preventive measures, joint replacement represents the principal option for relief of disability and dependency. ${ }^{1}$ The primary aim of this study was to conduct a full prospective psychological and social evaluation of joint replacement surgery to determine extent of change in a range of health indices assessing different aspects of life quality.

Evaluations of health care effectiveness typically use one of two measurement approaches, which we will refer to as "global" and "disease specific". A number of studies concerned with cost effectiveness have used global quality of life measures such as the Rosser-Kind Index ${ }^{2}$ and have demonstrated its sensitivity to change over time. ${ }^{3}$ While global measures such as the Rosser-Kind Index have the advantage of being brief to administer and may be used to compare the (cost) effectiveness of treatments for different conditions, they also have some limitations as measures of change in health status. For example, the Rosser-Kind does not distinguish between pain and different aspects of psychological well being, such as depression and anxiety. Similarly, the questions assessing disability combine a number of different activities within each disability category, making it difficult to assess where specific gains have been made. As a result, global measures such as the Rosser Kind may not provide information that is sufficiently detailed to direct clinical practice.

Disease specific measures are designed to assess outcomes with respect to a particular disease or a particular procedure. Disease specific measures include self report assessments such as the Harris Hip Score ${ }^{4}$ and the WOMAC. ${ }^{5}$ These measures are useful for the assessment of outcomes of interest to surgeons or physiotherapists, such as stiffness, or joint movement, but may not permit evaluation of changes in functional activities, which may be important indices of outcome to patients themselves, such as shopping, cooking or participation in social and leisure activities. Nor do such measures reliably assess psychological benefits. As different measures exist for different types of disease, comparison across disease types is difficult or impossible. For example, different measures are often used for clinical and physiotherapy assessment of the knee and hip, precluding comparison of relative (cost) effectiveness.

In this study we aim to provide data that complement those provided by evaluations based upon global or disease specific measures and aims to tackle some of the limitations posed by those measures. We aimed to conduct an evaluation that was capable of (a) providing data that was sufficiently specific to direct clinical practice and (b) providing patients and doctors with outcome data that relate to 
Table 1 Change in distress, pain, function, and informal care over the course of treatment ( $n=72)$. Data shown as mean $(S D)$

\begin{tabular}{|c|c|c|c|c|c|}
\hline & T1 Before surgery & $\begin{array}{l}\text { T2 } 3 \text { months after } \\
\text { surgery }\end{array}$ & $\begin{array}{l}\text { T3 } 9 \text { months after } \\
\text { surgery }\end{array}$ & $\begin{array}{l}\text { Percentage } \\
\text { change }{ }^{\star} 1-T 3\end{array}$ & $F(d f=2158) t$ \\
\hline \multicolumn{6}{|l|}{ Distress } \\
\hline Depression CES-D & $9.40(6.58)$ & $7.42(7.77)$ & $7.53(6.73)$ & -20 & $2.46 \mathrm{NS}$ \\
\hline Anxiety HADS & $9.29(4.93)$ & $7.39(4.91)$ & $7.76(4.65)$ & -17 & $4.96^{\star \star}$ \\
\hline \multicolumn{6}{|l|}{ Pain } \\
\hline Adjective checklist (McGill) & $22.26(9.34)$ & $4.94(5.38)$ & $3.72(3.72)$ & -83 & $195.69^{\star \star}$ \\
\hline Resting pain (VAS) & $21.38(27.16)$ & $4.21(9.48)$ & $5.28(13.450$ & -75 & $21.91^{\star \star}$ \\
\hline Worst pain (VAS) & $80.94(18.15)$ & $28.99(23.93)$ & $23.26(24.60)$ & -71 & $173.38^{\star \star}$ \\
\hline \multicolumn{6}{|l|}{ Function } \\
\hline Number of activities & $18.25(8.14)$ & $19.82(8.65)$ & $26.35(4.71)$ & +44 & $33.13^{\star \star}$ \\
\hline \multicolumn{6}{|l|}{ Informal support } \\
\hline $\begin{array}{l}\text { Number of tasks with which at least } \\
\text { one person is helping } \\
\text { Number of hours of informal }\end{array}$ & $2.01(2.67)$ & $1.33(1.63)$ & $1.07(1.20)$ & -53 & $5.46^{\star \star}$ \\
\hline support per week & $2.42(7.00)$ & $3.83(11.15)$ & $7.08(15.13)$ & +193 & $3.12^{\star \star}$ \\
\hline
\end{tabular}

$\star$ Percentage change was calculated as the mean difference between scores at T3 and T1 as a percentage of T1 scores. $†$ Repeated measures MANOVA. Within subjects effect of time is shown. ${ }^{\star} \mathrm{p}<0.05,{ }^{\star \star} \mathrm{p}<0.01$

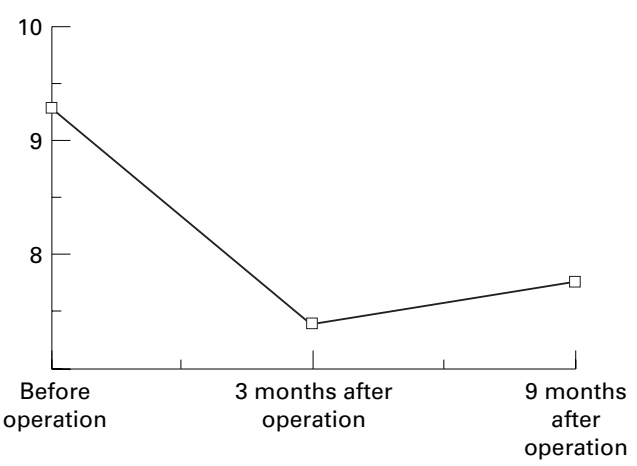

Figure 1 Change in anxiety.

outcomes of value to patients, permits realistic expectations, and allows for planning follow up services. Established psychological measures exist for the assessment of a range of health states. These measures are not disease specific and therefore permit comparison across disease types, as do global measures. However, because they each provide an assessment of a clearly defined and validated component of health status, they overcome some of the limitations of global measures such as the RosserKind. To our knowledge, no previous evaluation of joint replacement surgery has used independent measures of depression, anxiety, pain, and functional activity in the same study.

Specifically, we conducted an evaluation that included independent assessments of pain, anxiety, depression to determine what psychological benefits patients are likely to experience. We also sought to investigate, using

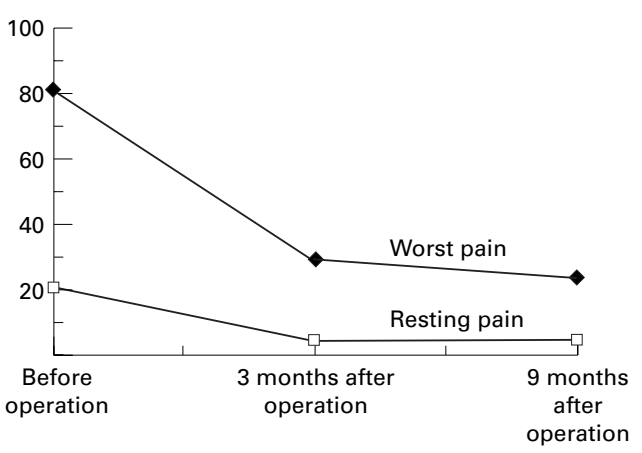

Figure 2 Change in pain. detailed home interviews, the extent to which patients initiated particular activities they had ceased before surgery. This information is not only of value to doctors and patients in guiding their expectations of surgery, but may inform rehabilitation professionals of specific areas requiring attention. Additionally, this study included an assessment of the impact of surgery on informal and formal care. Functional difficulties are a significant source of burden on informal carers in the community. ${ }^{6}$ The impact of surgery on modifying needs for assistance from informal and formal carers such as home helps is therefore an important concern for public health.

\section{Method}

PATIENTS

Patients having primary joint replacement surgery of the knee or hip at two centres in Scotland were recruited over a one year period. Patients undergoing revision surgery or replacement of joints other than the knee or hip, or who were receiving treatment for osteoarthritis $(n=176)$ were excluded from the study. Ethical approval was granted by the local medical ethics committee.

\section{PROCEDURE}

Before surgery, patients having primary surgery of the knee or hip were contacted initially by the bed managers in each of the two centres. At the same time as patients were contacted by the bed manager with an admission date, they were sent a letter informing them of the purpose of the study and inviting them to take part. They were then contacted by the interviewer who telephoned and arranged to visit them at home before admission. Six patients who fulfilled the recruitment criteria refused to take part in the study. Preoperative interviews were conducted, on average, 14 days before surgery. The purpose of the study was explained by the interviewer and patients were given an information sheet and asked to sign a consent form. Follow up interviews were arranged by the interviewer three months after surgery and nine months after surgery.

MEASURES

Depression was assessed by the Centre for Epidemiological Studies Depression Scale 
(CES-D). ${ }^{7}$ This measure has been used extensively among older populations ${ }^{8}$ and has been evaluated for possible confounds with psychosomatic symptoms. ${ }^{9}$ The CES-D is a 20 item self report measure. Respondents are asked how many times they have experienced each of 20 symptoms on a 4 point scale: 0 (rarely or none of the time), to 3 (most or all of the time). Scores are added up to give a possible range from $0-60$ with higher scores indicating more symptoms of depression. In this study, the reliability analyses of the scale produced Cronbach's $\alpha$ coefficients of $0.86,0.88$, and 0.91 at times one, two, and three respectively.

Anxiety was assessed by the anxiety subscale of the Hospital Anxiety and Depression Scale (HADS). ${ }^{10}$ This is a 7 item measure. Each item is scored on a 4 point scale (0-3) and summed to give a range from $0-21$. In this study, the Cronbach's $\alpha$ coefficient was $0.68,0.73$, and 0.73 at times one, two, and three respectively.

Three measures of pain were included to assess different aspects of pain experience. The first measure is a qualitative description of pain. ${ }^{11}$ This measure comprises a list of adjectives that describe the qualitative aspects of pain experience (for example, throbbing, aching, sharp). For each adjective the patient is asked to rate the extent to which they experience this form of pain. Scores are then summed to provide a total score. Additionally, two visual analogue scales were used to assess pain intensity. The first assessed resting pain, "How intense is your pain right now?" The second assessed patient perceptions of their pain at its worst, "How intense is your pain when it is at its worst?" Both visual analogue scales were $10 \mathrm{~cm}$ lines anchored at one end with the words "no pain" and at the other with the words "worst possible pain". The patient was asked by the interviewer to draw a slash across the line to indicate their response. Responses were scored in millimetres giving a possible range from 0 to 100 .

Functional activity was assessed by self report and recorded by the interviewer. A list of 32 activities was derived from existing scales and pilot interviews to comprehensively assess activities patients found difficult before surgery. The complete list of activities can be seen in table 3. The interviewer rated each activity on a dichotomous scale $(0=$ has not done this in past week/month) and ( $1=$ has done this in past week/month). All social and leisure activities appearing in the bottom portion of table 3 were assessed for a one month time period. Validity of non-observable activities such as shopping was established by asking the patient to give details of where and when they had last been shopping and to recall what they had purchased. For the present analysis, a total function score was obtained by adding scores for each activity to give a range from 0 to 32 . Increases in scores across time can therefore be interpreted as an increase in the number of different activities a person engages in. Reliability of the scale was high. Cronbach's $\alpha$ coefficient was 0.92 at time one, 0.91 at time two, and 0.91 at time three.

Two measures of informal support were calculated. Firstly, patients were asked if
KEY POINTS

- The assessment of health outcomes in service evaluation will benefit from the use of measures developed within psychology, which are capable of providing both information of value in comparing the (cost) effectiveness of different procedures and providing information sufficiently specific to direct clinical practice.

- Outcomes assessment that focuses on outcomes of importance to patients, their carers, and the community may enhance decision making in care of older people.

- Joint replacement surgery for patients with osteoarthritis of the knee and hip is effective in changing pain and functional activity. Gains in functional activity occurred in the period between three and nine months after surgery. One further direction for research may be to investigate sources of variability in outcomes.

anyone helped them with each of a list of 14 activities. This list was derived from previous research. ${ }^{12}{ }^{13}$ The complete list of items was; shopping, transportation, finances, laundry, legal matters, housework, preparing meals, nursing tasks, dressing/undressing, bathing, toileting, going to bed/getting up, eating, household repairs. A score of 1 was entered for each activity with which the patient received assistance from at least one person. A total score (number of activities supported) was generated by adding up the total number of activities with which the patient received assistance. The second measure of informal support was concerned with the amount of time informal supporters spent caring for the patient. The patient was asked about family members and friends who had cared for them during the past week. They were then asked how much time the person had spent helping them in the past week. Patients were helped to recall particular visits using memory probes. The scores reported in this paper are the sum of hours of informal support received from all informal sources in the one week period preceding each interview.

\section{Results}

\section{RESPONSE RATE}

One hundred and seven patients were recruited to the study. Six patients who fulfilled the research criteria refused to take part in the study. The response rates at three months and nine months follow up were satisfactory, at $79 \%$ and $67 \%$ respectively. At three month follow up two patients could not be traced and two had moved into residential care. Eighteen patients who were contacted by the interviewer did not complete questionnaires. An additional five patients did not complete the questionnaires at the three month follow up and five could not be traced, giving a final sample of 75 of whom complete measures were available for 72 patients. Those who withdrew from the study did not differ from those who completed all three interviews in terms of sex $\left(\chi^{2}=0.09\right.$, NS), type of joint 


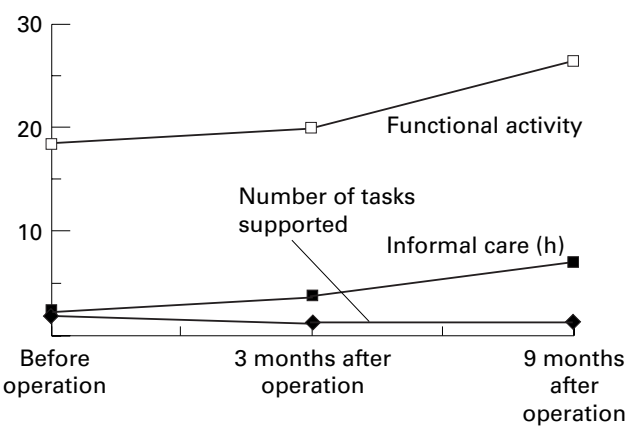

Figure 3 Change in functional activity and informal care.

$\left(\chi^{2}=0.71, \mathrm{NS}\right)$, age $(t=-1.20, \mathrm{NS})$, income $\left(\chi^{2}=-1.21, \mathrm{NS}\right)$, or on any of the preoperative measures of depression ( $t=-1.40, \mathrm{NS})$, anxiety ( $t=0.27, \mathrm{NS})$, functional activity $(t=$ $-1.22, \mathrm{NS})$, or pain $(t=-0.30,0.39 .1 .07$ respectively for three measures, all NS). This finding is consistent with that of Shaw et al. ${ }^{14}$

The final sample of 72 patients comprised 43 women and 29 men. Age ranged from 43 years to 85 years (mean (SD) 68.24 (9.05)). Average weekly income reported by the sample was $£ 109.82$ (SD £81.06). Average age of completing full time education was 15 years (SD 1.57). Fifty seven per cent of the sample owned a car and $26 \%$ lived alone. Forty three patients underwent surgery of the hip, and 31 underwent surgery of the knee. All patients had osteoarthritis.

OVERVIEW OF ANALYSES

Firstly, we analysed the group data to assess change from preoperative assessment to nine month follow up assessment using multivariate analysis of variance. Secondly, we conducted additional analyses to investigate change at the level of the individual patient. While analyses of group data are important in assessing the overall impact of an intervention on the population as a whole, individual within subject analyses are valuable in revealing the extent of variability in patients' responses to the intervention.

CHANGE OVER THE COURSE OF TREATMENT

Table 1 shows the mean (SD) scores obtained for each of the measures at the three time points. Multivariate analysis of variance was conducted to assess within subjects change over time. $F$ statistics are shown in table 1 .

Table 2 Percentage of patients experiencing improvement, no change, and deterioration of symptoms between the preoperative and 9 month interviews ${ }^{\star}$

\begin{tabular}{llll}
\hline & Improvement & No changet & Deterioration \\
\hline Distress & 59 & 6 & 35 \\
$\quad$ Depression CES-D & 59 & $6(3)$ & 35 \\
$\quad$ Anxiety HADS & 97 & 0 & 3 \\
Pain & 55 & $31(29)$ & 14 \\
$\quad$ Adjective checklist (McGill) & 97 & 0 & 3 \\
$\quad$ Resting pain (VAS) & & & \\
$\quad$ Worst pain (VAS & 45 & $27(19)$ & 28 \\
Informal support & 18 & $36(36)$ & 46 \\
$\quad$ Number of tasks with which at least one person & & & \\
$\quad$ was helping & & & \\
$\quad$ Number of hours of informal support per week & 18 & & \\
\hline
\end{tabular}

*All values are computed by subtracting nine month follow up scores from preoperative scores. †Figures in parentheses show the percentage of patients reporting no symptoms either before or after surgery.
Scores at nine month follow up were significantly different at the $0.1 \%$ level from preoperative scores for all measures except depression. Patients also reported significant gains in activities over time. The findings for informal support demonstrated opposing effects. While patients reported need for assistance with fewer tasks after surgery, they also reported an increase in the number of hours informal support they received after surgery.

CHANGE DURING SPECIFIC PHASES OF FOLLOW UP Figures 1 to 3 illustrate the patterns of changing distress, pain, function, and support over the course of the study. Inspection of the figures shows that reduction in distress and pain was most dramatic in the period between the preoperative assessment and the three month follow up. Muliple one way analyses of variance comparing within subject scores at times two and three showed that no significant change in these measures occurred between the three month and nine month follow ups. However, figure 3 shows a different pattern of findings. There was no significant change in functional activity between the preoperative and three month assessment. However, patients continued to acquire new activities between the three month postoperative assessment and the nine month postoperative assessment $(F=46.80, \mathrm{p}<0.01)$.

\section{INDIVIDUAL ANALYSES OF CHANGES IN PAIN AND} DISTRESS

Data based on averages do not permit examination of individual patients' experience. This is because changes in a positive and negative direction are liable to cancel each other out. We therefore calculated the difference between nine month follow up scores and preoperative scores for each individual patient. Table 2 shows the percentages of patients demonstrating an improvement, no change or deterioration on each measure. A third of patients reported more symptoms of depression and anxiety at follow up. Very small numbers of patients reported increases in pain after surgery (3-14\% across measures). It is important to note that a large proportion of patients were pain free on the resting pain measure before surgery. The apparently large percentage of patients demonstrating no change on this measure is therefore because of these patients reporting no pain either before or after surgery. Just under a third of patients reported a deterioration (help needed with more tasks) in task assistance and nearly half reported more hours of informal support.

SPECIFIC ACTIVITY GAINS AND LOSSES

To assess the specific gains made by patients and their residual levels of function after surgery, we conducted additional within subject analyses to calculate (a) the percentage of patients not performing activities before or after surgery, (b) the percentage of patients performing activities before surgery but losing them over time, (c) the percentage of patients recovering an activity they were performing before surgery, and (d) the percentage of 
patients acquiring new activities that they were not performing before surgery. Table 3 gives the results. Losses over time were small and affected $1-10 \%$ of patients. Four per cent of patients lost the ability to get down on the floor to pick something up, $7 \%$ no longer used a bath independently, $10 \%$ ceased heavy cleaning. Five per cent of patients stopped driving after surgery and $8 \%$ stopped using the bus. To determine the significance of these losses, the number of patients losing activities was compared against an expected frequency of zero. These analyses involved only patients performing activities before surgery. Significant effects were obtained for the numbers of patients losing light cleaning $\left(\chi^{2}=8.14, \mathrm{df}=1, \mathrm{p}<0.01\right)$, heavy cleaning $\left(\chi^{2}=49.92, \mathrm{p}<0.01\right)$, shopping $\left(\chi^{2}=19.56, \mathrm{p}<0.01\right)$, driving $\left(\chi^{2}=41.98\right.$, $\mathrm{p}<0.01)$, use of the bus $\left(\chi^{2}=95.36, \mathrm{p}<0.01\right)$, the ability to pick something up from the floor $\left(\chi^{2}=29.17, \mathrm{p}<0.01\right)$, and use of the bath $\left(\chi^{2}=\right.$ $5.79, \mathrm{p}<0.05)$. In contrast, gains in newly acquired activities ranged from $1-70 \%$ over time. In terms of mobility the most frequently acquired activity was walking up and down hills (road hills) $(54 \%)$. Forty one per cent of patients acquired the ability to pick something up from the floor. Substantial gains were made for all domestic activities, the most common being shopping for non-food items ( $49 \%$ of patients). Twenty eight per cent of patients acquired the ability to use the bus after surgery. Increases were observed in all social and leisure activities, with $36 \%$ of patients acquiring community activity involvement, $43 \%$ going out socially, and $51 \%$ acquiring active pastimes.

FORMAL SERVICES

We assessed change in receipt of five community care services; home help, district nurse, meals on wheels, laundry service, and day centre attendance. Overall, we observed increases in use of services over the course of the follow up. Preoperatively, six patients had a home help $(8 \%)$. At the nine month follow up, 17 patients $(21 \%)$ had a home help. No patient discontinued the home help service as a result of surgery but 11 patients gained a home help. No patient was receiving visits from a district nurse, or using a laundry service either before or after surgery. Before surgery, no patient was receiving meals on wheels, but two patients obtained this service during the study. One patient attended a day centre before surgery, and two reported using this service after surgery. As the increase in the use of home help services was unexpected, we conducted a further telephone follow up 18 months after surgery. Six patients were found to have discontinued the service at this time, 11 were still in receipt of home help services.

TYPES OF INFORMAL SUPPORT

Examination of the types of help provided by informal supporters showed that over the period between preoperative assessment and nine month follow up, increases in assistance occurred for four types of care. Increases in assistance were observed for laundry, legal matters, using the toilet, and going to bed. For
Table 3 Percentage of patients not performing, losing, recovering or acquiring activities over the course of treatment, preoperative to 9 month follow up $(n=72)$

\begin{tabular}{|c|c|c|c|c|}
\hline & $\begin{array}{l}\text { Does not } \\
\text { perform } \\
\text { activity }^{*}\end{array}$ & $\begin{array}{l}\text { Loses } \\
\text { activityt }\end{array}$ & $\begin{array}{l}\text { Recovers } \\
\text { activity } \neq\end{array}$ & $\begin{array}{l}\text { Acquires } \\
\text { activityS }\end{array}$ \\
\hline \multicolumn{5}{|l|}{ Activity } \\
\hline Stand unsupported & 0 & 0 & 80 & 20 \\
\hline Climb stairs & 3 & 1 & 60 & 36 \\
\hline Walk across room & 0 & 0 & 99 & 1 \\
\hline Walk out of doors & 0 & 1 & 76 & 23 \\
\hline Walk up/down hills & 3 & 1 & 42 & 54 \\
\hline Get in and out of bed & 0 & 1 & 88 & 11 \\
\hline $\begin{array}{l}\text { Get down to pick } \\
\text { something up }\end{array}$ & 11 & 4 & 44 & 41 \\
\hline \multicolumn{4}{|l|}{ Get in and out of } & 11 \\
\hline Get in and out of bath & 11 & 7 & 70 & 12 \\
\hline Wash body all over & 0 & 0 & 89 & 11 \\
\hline Put on shoes & 0 & 0 & 84 & 16 \\
\hline Dress & 0 & 0 & 88 & 12 \\
\hline Fasten clothing & 0 & 0 & 89 & 11 \\
\hline Make a hot drink & 1 & 1 & 78 & 18 \\
\hline Make a meal & 6 & 1 & 69 & 24 \\
\hline \multicolumn{5}{|l|}{ Carry a hot } \\
\hline drink/meal & 4 & 1 & 65 & 30 \\
\hline Light cleaning & 7 & 4 & 56 & 33 \\
\hline Heavy cleaning & 16 & 10 & 39 & 35 \\
\hline Laundry & 16 & 2 & 53 & 29 \\
\hline Shop for food & 5 & 5 & 64 & 26 \\
\hline \multicolumn{5}{|l|}{ Shop for non-food } \\
\hline items & 6 & 1 & 44 & 49 \\
\hline Get in/out of a car & 1 & 5 & 78 & 16 \\
\hline Drive a car & 55 & 5 & 33 & 7 \\
\hline Use the bus & 28 & 8 & 36 & 28 \\
\hline Go into town & 4 & 1 & 70 & 25 \\
\hline Indoor hobbies & 0 & 0 & 88 & 12 \\
\hline Community activities & 26 & 2 & 36 & 36 \\
\hline Have visitors & 0 & 1 & 71 & 28 \\
\hline Visit friends/relatives & 3 & 2 & 63 & 32 \\
\hline Go out socially & 10 & 3 & 44 & 43 \\
\hline Go away from home & 0 & 1 & 29 & 70 \\
\hline Active pastimes & 28 & 1 & 20 & 51 \\
\hline
\end{tabular}

$\star$ Does not perform activity before surgery and does not perform activity at nine month follow up. +Performs activity before surgery but does not perform activity at follow up. $\ddagger$ Performs activity before and after surgery. \Does not perform activity before surgery but performs activity after surgery.

all other types of care; shopping, transport, finances, housework, meal preparation, dressing, bathing, eating, and household maintenance reductions in support were reported.

FACTORS ASSOCIATED WITH ABILITY TO BENEFIT As the within subject analyses had revealed considerable individual variability in change in outcomes, we examined the effects of four moderator variables on change over time; whether patients had knee or hip joint replacement, sex, age and income. Preliminary analyses were conducted to assess the independence of the proposed moderator variables. One association was obtained; women were more likely to have an income that fell below the median for the sample as a whole $(63 \%$ of women compared with $23 \%$ of men) and this effect was significant $\left(\chi^{2}=12.05, \mathrm{p}<0.01\right)$. All other moderator variables were independent of each other. Continuous variables were recoded into categorical variables by dividing at the median into high and low. The presence of an interaction of the moderator with time indicates that the extent of change observed over the course of treatment is a function of the moderator variable. The results of the analysis for each outcome variable produced no significant interactions of time with the moderator variables, suggesting that change in anxiety, depression, pain, functional activity, and 
informal care was independent of a patient's age, income, sex or knee versus hip joint.

\section{Discussion}

The aim of this study was to demonstrate the value of using independent assessments of different aspects of health. Overall, patients were found to make gains in anxiety reduction, pain reduction, and increased functional activity. While change in pain and anxiety occurred principally during the first three months after surgery, activity gains were greatest in the period between the three and nine month evaluations. Although pain was reduced and activity levels increased, levels of depression were not significantly changed over the course of the study. This suggests that depressed mood among patients with osteoarthritis is not directly related to levels of physical impairment and not amenable to change by surgery. Social and personal factors are more likely to determine levels of depressed mood.

Our examination of change in functional activities demonstrates where gains and losses are made after surgery. Substantial gains in functional activity occurred between the three month and nine month assessments. Patients made gains particularly in outdoor mobility and difficult movements such as getting down to pick something up. Non-essential shopping and active pastimes were also gained by half of the patients. Specific losses of activity as a result of surgery were small. However, the findings that $5 \%$ of patients stopped driving after surgery and $8 \%$ stopped using the bus suggests that attention in rehabilitation might be given to these activities. It may be that during initial recovery, patients lose confidence in performing these activities and fail to regain them over time. It may also be possible that patients in fact walk more after surgery, instead of using the bus. It is likely that the other losses we observed, which principally affected cleaning and shopping, are accounted for by changes in formal help. Seventeen patients received home help services as a result of undergoing surgery. It is noteworthy that the provision of home helps seemed to substitute for activities that the patient was performing themself before surgery. Home helps did not seem to substitute for activities that were previously performed by informal carers.

As expected, patients received assistance with fewer activities after surgery, indicating that treatment was effective in reducing this aspect of burden on carers. Continued assistance was required by some patients with laundry. Qualitative accounts provided to the interviewer indicated that this was because hanging washing on a line requires two free hands and the risk of loss of balance. Some patients also reported difficulty reaching low automatic machine doors. It is curious that a small percentage of patients reported continued assistance with toileting and going to bed at nine month follow up. It may be that patients continue to experience anxiety about sitting on low toilet seats, are unable to get up from the toilet unaided, and experience concern about the correct way to lie in bed. At initial discharge, patients are given specific instruction in this regard, which they may continue to attend to and/or worry about after discharge. It may be useful to ensure that attention is given to these activities at discharge and home assessment to relieve anxiety.

Although surgery seemed to be effective in reducing need for task assistance in the majority of patients, paradoxically the number of hours of informal support received by patients increased over the period of follow up. While it might be expected that patients would receive increased support during hospitalisation and convalescence, the data showed that this effect was not restricted to the period immediately after surgery, as the number of hours of support continued to rise until the nine month follow up. We also noted increases in the use of formal services that were maintained up to 18 months after surgery. Additionally, during the home visits, several patients commented that as a result of surgery they had been provided with useful equipment such as helping hands and bath boards, which assisted their functional activity and would have been valuable before surgery. While it is the case that such devices are provided in part, at least, to prevent patients making movements that might threaten the integrity of their replaced joint, this finding demonstrates, none the less, that entry to surgery operated as a gateway to a range of formal and informal supports, and particularly to increased support from friends and family. It may be that treatment served to legitimise patients' need for support in pursuing functional activity.

The within subject analyses demonstrated that there is considerable variability in the effectiveness of joint replacement surgery. This might be attributable to (a) variations in the surgical and rehabilitation procedures or (b) to psychological factors such as patients' beliefs about their condition or its treatment. ${ }^{15}$ This study suggested that variability was not a result of knee compared with hip replacement, sex, age or income, although a larger scale replication of this finding would be desirable.

The comprehensive prospective evaluation of joint replacement surgery provided here has demonstrated the value of using independent assessments that can provide doctors and patients with information that enables them to develop realistic expectations of surgery and can guide rehabilitation professionals to specific areas requiring attention. The data provided here may be used in the process of decision making about surgery and the findings concerning lost activities may be of interest to rehabilitation specialists and by surgeons during discharge visits. Finally, the use of the measures reported here for health care evaluation is to be encouraged, both with respect to joint replacement and other procedures.

We would like to thank Lindsay McGregor, Mary-Frances Morris, Gail Johnston, Jean Orme, Mark Elvin for their assistance in conducting the study and all the patients who gave up their time to participate.

Funding: the research reported in this paper was supported by the MRC (G106/536). 
1 Verbrugge L M. Disability. Rheum Dis Clin North Am 1990;741-61.

The measurement of hospital output. Int $\mathcal{F}$ Epidemiology1972:1:361-8.

3 Norman-Taylor F H, Palmer C R, Villar R N. Quality of life improvement compared after hip and knee replacement. $f$ Bone foint Surg 1996;78B:74-7.

4 Harris W H. The Harris Hip Score. $f$ Bone foint Surg1969: 51A:737-55.

5 5Bellamy N. WOMAC. Semin Arthritis Rheum 1989:18 (suppl 2):14-17.

6 Orbell S. Informal care in social context: A social psychological analysis of participation, impact and intervention in care of the elderly. Psychology and Health 1996;11:155-78.

7 Radloff L S. The CES-D scale: A self-report depression scale for research in the general population. Applied Psychological Measurement 1977;1:385-401.

8 Gatz M, Hurwicz M-L. Are old people more depressed? Cross-sectional data on center for epidemiological studies depression scale factors. Psychol Aging 1990;5:284-90.
9 Coyle C, Roberge J J. The psychometric properties of the center for epidemiological studies depression scale CES-D) when used with adults with physical disabilities. Psychology and

10 Zigmond A S, Snaith R P. The Hospital Anxiety and Depression Scale. Acta Psychiatr Scand 1983;67:361-70.

11 Melzack R. The Short-form McGill Pain Questionnaire. Pain 1987;30:191-7.

12 Orbell S, Gillies B, Hopkins N. A survey of people providing informal care to an elderly person in Tayside. Report to Tayside Health Board. Available from the first author, Department of Psychology, University of Sheffield.

13 Orbell S, Gillies B, Hopkins N. Measuring the impact of informal caring fournal of Community and Applied Social Psychology 1993;3:149-63.

14 Shaw W S, Cronan T A, Christie M D. Predictors of attrition in health intervention research among older subjects with osteoarthritis. Health Psychol 1994;13:421-31.

15 Orbell S, Johnston M, Rowley D, et al. Cognitive representations of illness and functional and affective adjustment following surgery for osteoarthritis. Soc Sci Med (in press). 